\title{
A green version of Haeckel's rule
}

\section{Peter Nick}

Received: 21 May 2010 / Accepted: 21 May 2010

(C) Springer-Verlag 2010

During individual development, many organisms recapitulate their evolutionary past - this core element of Haeckel's biogenetical rule, despite some debate on its overgeneralization by Haeckel, has been confirmed in numerous examples. For instance, the male gametophytes of angiosperms undergo a developmental program that repeats many cellular aspects from gametophytic development of the earliest land plants, the mosses, such as asymmetric division, highly polarized tip growth, and directional movement of plastids and mitochondria. The machinery of plastid division in mosses differs in both molecular composition and functional aspects from that found in vegetative cells of higher plants (Suppanz et al. 2007). Chloroplast division depends on prokaryotic tubulin homologues, the FtsZ proteins. Similar to mitochondria, chloroplasts have lost most of their genetic autonomy by transfer of genes into the nuclear genome, which means that most proteins have to be imported from the cytoplasm into the chloroplast, including FtsZ and other components of plastid division. When FtsZ was specifically knocked down in moss Physcomitrella patens (Strepp et al. 1998), vermiform and giant chloroplasts were produced, demonstrating that FtsZ was essential for plastid division. However, the situation has turned out to be more complex in the meantime. The FtsZ proteins are encoded by a small gene family, whereby some members (that have been lost during land-plant evolution) are localized in the cytoplasm and

\section{P. Nick $(\bowtie)$}

Molekulare Zellbiologie, Botanisches Institut,

Karlsruhe Institute of Technology,

Kaiserstraße 2,

76131 Karlsruhe, Germany

e-mail: peter.nick@bio.uni-karlsruhe.de possibly interact with the cytoskeleton of the "host cell" (Kiessling et al. 2004). Whereas the core function of these FtsZ isotypes seems to be in plastid division, some of them appear to have adopted additional functions that are related to plastid morphogenesis, especially the formation of extensions towards other plastids, so called stromules (Suppanz et al. 2007). The function of these FtsZ isotypes has remained enigmatic as well as the "disappearance" of some of them during cormophyte evolution. One approach to search for evolutionary missing links is to investigate early development, making use of Haeckel's rule. In this context, the behavior of plastids in the male gametophyte is highly interesting, but has remained elusive so far. Using GFP-fusions of the stroma-localized Arabidopsis thaliana FtsZ1 driven by their native promoter, Fujiwara et al. (2010) in the current issue have been able for the first time to follow the behavior of plastids in developing pollen tubes in the homologous system. This first glimpse reveals a few surprises.

Whereas in vegetative cells of mature pollen plastids were heterogeneous in shape, either round to ellipsoidal, the plastids of pollen tubes exhibited remarkable tubulation, stromule extension, and back-and-forth movement along the direction of tube growth. Interestingly, plastid division ceases with pollen maturation and seems to be replaced by dramatic shape alterations during their transport in the tube. These changes in shape correlate with the activation of cytoplasmic streaming suggesting interaction of the plastidbased machinery responsible for remodeling of the envelope and the cytoplasmic cytoskeleton. The full significance of these novel and exciting observations is still far from being completely understood, but it seems already now that this work allows unexpected insights into the mysterious past of present-day plastids. 


\section{References}

Makoto Fujiwara, Haruki Hashimoto, Yusuke Kazama, Tomonari Hirano, Yasushi Yoshioka, Seishiro Aoki, Naoki Sato, Ryuuichi Itoh, Tomoko Abe (2010) Dynamic morphologies of pollen plastids visualised by vegetative-specific FtsZ1-GFP in Arabidopsis thaliana. doi:10.1007/s00709-010-0119-7

Kiessling J, Martin A, Gremillon L, Rensing SA, Nick P, Sarnighausen E, Decker EL, Reski R (2004) Dual targeting of plastid division protein FtsZ to chloroplasts and the cytoplasm. EMBO Rep 5: 889-894

Strepp R, Scholz S, Kruse S, Speth V, Reski R (1998) Plant nuclear gene knockout reveals a role in plastid division for the homolog of the bacterial cell division protein FtsZ, an ancestral tubulin. Proc Natl Acad Sci USA 95:4368-4373

Suppanz I, Sarnighausen E, Reski R (2007) An integrated physiological and genetic approach to the dynamics of FtsZ targeting and organisation in a moss, Physcomitrella patens. Protoplasma 232: $1-9$ 